\title{
Fast/Major and Slow/Minor pairings in J.S. Bach's Well Tempered Clavier Books 1 and 2
}

\author{
KATELYN HORN[1] \\ Music Theory, The Ohio State University \\ EUGENIA COSTA-GIOMI \\ Music and Human Learning, The University of Texas at Austin
}

\begin{abstract}
Some studies have found that subjects give higher preference ratings and clearer emotional descriptions to pieces with the tempo/mode pairs fast/major and slow/minor. If these findings are true, one might expect to find a predominance of fast/major and slow/minor pairings in musical literature. This study looks for just such a trend in the canonical work of J.S. Bach's Well Tempered Clavier. Books 1 and 2 together contain 24 Major Preludes, 24 Minor Preludes, 24 Major Fugues and 24 Minor Fugues. We use four different measurements of fast/slow tempo based both on elements within the musical notation as well as the interpretive markings of four different music editors: 1. Attacks Per Beat (APB) - measures the number of subdivisions of each piece 2. Attacks Per Minute (APM) - is the APB of a piece multiplied by the editor's metronome marking 3. Time Signature Distribution and 4 . Editor's Tempo Distribution. We find a significant amount of fast/major and slow/minor pairings in Book 1 but not in Book 2.
\end{abstract}

Submitted 2011 August 25; accepted 2012 February 2.

KEYWORDS: music, minor mode, minor key, tempo, sad, J. S. Bach

TEMPO/mode pairings have often been studied in the context of musical preference and happy/sad judgments. There have been studies on the role of tempo and modality in the perception of happy vs. sad music (Hevner, 1935, 1937; Desroches, 1997; Dalla Bella, 2001; Gagnon et al., 2003), the role of tempo and modality in musical preference (LeBlanc 1983 and 1988, Flowers 1988), and the effect of tempo and mode on mood and arousal (Husain et al 2002). These studies have shown that tempo is considered the most significant musical dimension when subjects are asked to determine the emotional character of a piece or to make a preferential judgment between pieces. Mode also affects a person's musical preference and their perception of the emotional character of a piece, but the importance of mode tends to be secondary to that of tempo. Several studies (Flowers, 1998; LeBlanc, 1983, 1988) found fast tempo in particular to be a distinctive feature of preferred musical selections. This finding was bolstered by another study (Husain, 2002) which found that fast tempo also seems to increase arousal in listeners.

The primary focus of these studies was to understand musical preference or emotional character. In the process, though, they also found some interesting relationships between tempo and mode. Hevner found that music in a major mode (1935) or with a fast tempo (1937) is often labeled happy and that music in a minor mode (1935) or with a slow tempo (1937) is often labeled sad. In a more recent study (Gagnon, 2003), the happy/sad distinction was strongest in music that paired fast tempo with major mode and slow tempo with minor mode. Husain (2002) found [in a small subtest within her experiment] that listeners considered fast/major tunes most enjoyable and slow/minor tunes second most enjoyable out of the four possible tempo/mode pairings (fast/major, fast/minor, slow/major, slow/minor). On the other hand, Flowers (1988) found that listeners rated the fast/major tune first and the slow/minor tune last on a preference scale. In all of these studies, whether the focus was on emotional clarity, personal preference, or attention arousal, there seems to be something special about the particular tempo/mode pairs of fast $/$ major and slow $/$ minor.

Post and Huron (2009) looked directly at these tempo/mode pairings within Western classical music of the common practice era. They based their research on work done in speech prosody, which also 
shows an association between slow tempo and sad emotion. Their hypothesis was that since slow tempo is generally associated with sad emotion in speech and the minor mode is generally associated with sad emotion in Western classical music, then one would expect to find a strong association between slow tempo and minor mode within Western classical music. They did a survey of the three periods of the common practice era (Baroque, Classical, and Romantic) and found that such an association did exist but only in the Baroque and Classical periods.

Our study pursues a similar hypothesis to Post and Huron's study and is consistent with their findings. Instead of a broad survey of the literature, however, we chose to limit our sample to J.S. Bach's Well Tempered Clavier, which consists of two books of 24 preludes and fugues. Though hardly representative of Western classical music as a whole, J.S. Bach's Well Tempered Clavier is arguably one of the most influential musical texts within the field. Bach systematically works through all 12 keys in each book writing a Prelude and Fugue for each of the major keys and each of the minor keys. The result - a neatly arranged set of 48 minor pieces and 48 major pieces within the same musical genre - is a nice sample for a study which wishes to look for significant tempo/mode pairings. Furthermore, the Well Tempered Clavier has long been considered a critical component of any pianist's repertoire and is one of the most ubiquitous models used in basic composition and part-writing classes. It therefore holds a venerable place in the canon of Western classical music as part of the foundation of the common practice era.

The notion of tempo is a central and important element of music. When trying to define tempo, however, the concept turns out to be rather slippery. One might simply rely on the words people use to describe tempo, such as fast, slow or moderate - in various languages, such as the Italian terms, allegro, adagio, and moderato. The history of music is replete with examples where such terms are regarded as highly ambiguous, and not especially useful. For example, "a walking pace" might seem clear, except that the Italian term for this ("andante") has been interpreted dramatically differently in different periods. It is widely thought by musicologists that andante became slower in the late 19th and early 20th centuries.

The invention of the metronome by Johann Mälzel in the first half of the 19th century was widely hailed as providing a way to clearly specify the tempo. Although metronome markings may allow performers to replicate a precise tempo, the metronome markings themselves tell us little about the sense of speed or feeling of musical rapidity. It is problematic to claim that 80 beats per minute is faster than 70 beats per minute without considering such aspects as the texture, density, and harmonic rhythm of the work.

Even when the beat rates are identical, the manner of beat subdivision may influence how the tempo is perceived. So a $6 / 8$ passage may be perceived as faster than a $2 / 4$ passage even when the beat rate is identical. Furthermore, expressive performances often employ rubato, which can cause the perceived tempo to vary widely throughout the course of a piece. (See also London, 2004, 2011)

Given the complexities involved, one might simply despair and conclude that tempo is ultimately enigmatic and impossible to characterize. While recognizing the slipperiness of this theoretical concept, we might nevertheless recognize that it should be possible to estimate the tempo under some circumstances. Hence, for example, although tempo terms are ambiguous, one might still reasonably assume that a passage marked "presto" might well be perceived as faster than a passage marked "allegro," that a passage containing a metronome marking of $80 \mathrm{bpm}$ might well be perceived as faster than a passage marked 70 bpm, and that a passage containing 3.8 note onsets per second will be experienced as "faster" than a passage containing 3.1 note onsets per second. definitions:

For the purposes of this study, we will attempt to estimate tempo using four operational

(1) The number of attacks per beat or APB,

(2) The rate of attacks per minute or APM,

(3) Time signature

and

(4) Italian tempo descriptions such as allegro and andante.

While none of these can be regarded as an accurate way of characterizing tempo, we hope that the results for these different estimates might converge on similar answers, and so help us address our hypothesis concerning the relationship between mode and tempo. 


\section{METHOD}

\section{Materials}

Although Bach did not give tempo markings for the vast majority of the preludes and fugues, there is a pool of later editions with tempo markings and metronome suggestions added. These tempo and metronome markings can be somewhat problematic, however, as already discussed. Three particular problems that arise are (1) the vagaries of language used in describing tempos, (2) how to determine the beat level, and (3) the manner in which subdivisions of the beat affect tempo perception. We attempted to devise measurements that dealt with as many of these aspects of tempo perception as possible.

One way to measure the tempo of a piece would be to have a group of listeners categorize the tempo of a set of representative performances. However, for logistical reasons, we chose to limit ourselves to data from various publications of the musical text and did not consult performances or recruit listeners. We developed four separate measurements, two of which were based on features of the musical notation and two of which were based on editorial interpretations. We used an Urtext copy of Bach's music from the publisher G. Henle (Ed. Irmer, 1950) to collect the data on the notational features of the music. The Henle Urtext is nearly identical to a more recent Urtext edition, the Neue Bach Ausgabe, especially concerning notated rhythms and time-signatures. For the editorial interpretations, we looked at the editions of Donald Francis Tovey (1924), Bruno Mugellini (1936), Hans Bischoff (1942), and Bela Bartok (1964). All the editors gave a tempo and/or character description to each piece, but only Bartok, Bischoff and Mugellini assigned metronome markings. Bartok did not give any markings for two major Preludes and Fugues from Book 1 and three major and three minor Preludes and Fugues from Book 2.

\section{Measures}

The first measurement we took from the musical notation of the Urtext version was the average number of attacks per beat, or APB. The number of attacks in each measure was determined by manually counting the number of attacks in the surface rhythm created by the rhythmic sum of all the voices. This is demonstrated in Figure 1a and 1b. The rhythms of the two voices shown in Figure 1a can be combined to create the surface rhythm shown in Figure 1b. The number of attacks in the resulting surface rhythm, then, is 13 .

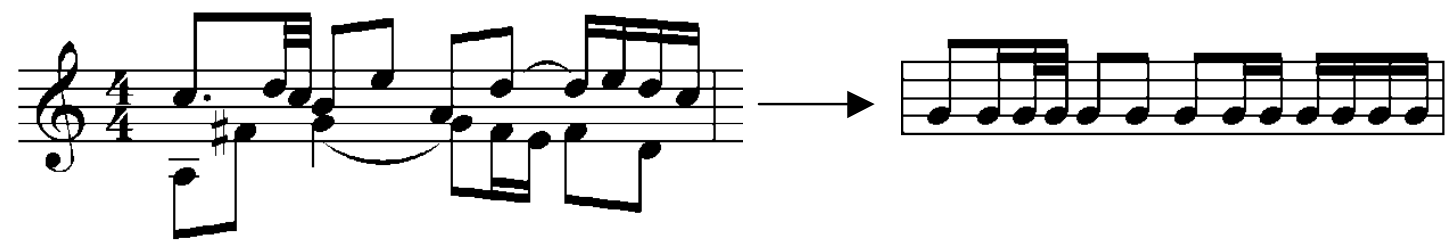

Fig. 1a. Excerpt from Book 1 Fugue no. 1 in C Maj.

Fig. 1b. Rhythmic sum of the two voices in 1a equals 13 attacks.

After calculating the number of attacks in each piece we divided that number by the total number of beats in the piece. The result is the average number of subdivisions for each beat in that piece. For example; if a piece has 951 attacks, 70 measures and has a 4/4 meter, the resulting APB is 3.40 (951 attacks $/ 280$ beats). The higher the APB the more subdivisions a piece has and the more rapid we assume it is perceived to be. In cases where a piece was in compound meter, according to common practice, we calculated the APB with the larger note-value as the beat. Thus if a piece was in $6 / 8$ we considered the dotted-quarter note to be the beat and counted 2 beats per measure. By treating an eighth note in $6 / 8$ as $50 \%$ faster than an $8^{\text {th }}$ note in $2 / 4$, we are giving primacy to the tactus or beat level. This, if the beat-rate is similar, then $6 / 8$ will tend to sound faster. When calculating this and other measurements, two pieces were omitted from book 2 (one major prelude and one major fugue) because the time signature changed in the middle of the piece. Most of the calculations for book 2, then, were based on 46 rather than 48 pieces.

The second measurement we took was the average number of attacks per minute, or APM, of each piece. This was done by multiplying a given piece's APB (attacks per beat) by an editor's metronome 
marking which was given in beats per minute, or BPM. The more attacks per minute a piece has, the faster it is considered to be. We did this for each editor who gave metronome markings (Bartok, 1964; Bischoff, 1942; Mugellini, 1936). In a few cases, the editors gave a metronome marking for a note value other than indicated by Bach's time signature. In such cases, we recalculated the APB so that the beat values of both the APB and the BPM matched. For example, if a piece had a $4 / 4$ meter we calculated the APB with the quarter-note as the beat, thus counting four beats per measure. But if one of the editors gave a metronome marking for the eighth note rather than a quarter-note, we recalculated the APB with the eighth-note as the beat, now counting eight beats per measure. The important factor was that the beat values agreed [2].

The third measurement we took was the distribution of time signatures. The reasoning for this was that while a piece in $9 / 8$ and a piece in $3 / 2$ may have the same number of attacks per beat, the piece in $3 / 2$ will most likely be regarded by the music reader as slower because the note values being used are larger/longer than those for the piece in 9/8. In fact, one editor of Bach's WTC (Palmer, 1981) states that a piece in $2 / 2$ indicates two slow beats rather than two fast beats. The significant number here is the bottom number of the time signature, because that tells us what note value gets the beat. By this standard, Bach uses four different time signatures: $-/ 2,-/ 4,-/ 8,-/ 16$. We looked at what percentage of all the pieces of each time signature was major and what percentage was minor.

The fourth and final measurement we took was the distribution of tempo descriptions given by all four editors. To do this we classified each description into one of three categories; $1=$ fast, $2=$ moderate/unclear, 3 = slow. Our definitions of each category were as follows: vivace/veloce, allegro and allegretto as fast; grave, lento/lentamente, largo/larghetto/largamente, adagio and andante as slow; and moderato, andantino and character descriptions such as grazioso, tranquillo, sostenuto etc. as moderate/unclear. We then looked at what percentage of the fast descriptions was given to major or minor pieces and what percentage of slow descriptions was given to major or minor pieces. We omitted the moderate/unclear category, as it was not relevant to this study.

After determining the APB for each piece and the APM for each editor's metronome marking, we then calculated the average of each for different combinations of piece (prelude and fugue) and book (book 1 and book 2), always comparing the major-mode results to the minor-mode results. In order to determine whether or not the difference between the major-mode averages and minor-mode averages was significant, we used Analyses of Variance (ANOVAs) to analyze the data. When analyzing the distribution of time signatures and tempo markings, it was sufficient to find the percentage of the whole. The different sets we chose to look at were as follows:

1. Separated book and piece type, thus strictly comparing major preludes from book 1 to minor preludes from book 1 etc.

2. Combined books but separated piece type, thus comparing major fugues from both books to minor Fugues from both books, etc.

3. Combined piece types but separated books, thus comparing all major pieces from book 2 to all minor pieces from book 2 , etc.

4. Combined books and piece type, thus comparing all major pieces of both books to all minor pieces of both books.

We used all of these combinations to analyze the data from all four of the measurements, but found the third set (combined pieces/separated books) to be the most illuminating and thus will focus the results section of this paper on those data.

\section{RESULTS}

Given that we have exhausted the data and given the negligible moral repercussions of committing a type 1 error, for the purposes of this study we a priori set our alpha level at 0.1 . The results of all four measurements were consistent in showing fast/major and slow/minor pairings to be favored in book 1 but not in book 2 .

As shown in Table 1, within book 1 all the major mode pieces had a higher APB than minor mode pieces. This difference was significant, $F(1,47)=7.79, p=.008$. In book 2 , major pieces also had a higher $\mathrm{APB}$ than minor pieces but the difference in this case was not significant. 
Table 1. Average Attacks per Beat (APB) for major and minor pieces of books 1 and 2.

\begin{tabular}{|l|l|l|l|l|}
\hline Book & Major & Stand. Dev. & Minor & Stand. Dev. \\
\hline $\mathbf{1}$ & $\mathbf{4 . 4 0}$ & 1.29 & $\mathbf{3 . 4 0}$ & 1.21 \\
\hline $\mathbf{2}$ & $\mathbf{3 . 6 7}$ & 0.88 & $\mathbf{3 . 9 8}$ & 0.85 \\
\hline
\end{tabular}

The average APM as indicated by all three editors for major and minor pieces of books 1 and 2 are shown in Table 2. In order to avoid violating the assumption of independence we averaged the 3 APM scores for each piece before running the ANOVA. Here we see very similar results to those found for the APB measurement. Within book 1, the average APM for a major mode piece was significantly higher than the average APM for a minor mode piece $F(1,43)=16.89, p=0.000$. We see again, however, that this is not the case for book 2. In fact, there is hardly any difference at all between the average APMs of major and minor pieces in book 2 .

Table 2. Average Attacks per Minute (APM) for major and minor pieces of books 1 and 2 with combined editors.

\begin{tabular}{|l|l|l|l|l|}
\hline Book & Major & Stand. Dev. & Minor & Stand. Dev. \\
\hline $\mathbf{1}$ & $\mathbf{3 8 5 . 7}$ & 112.5 & $\mathbf{2 4 6 . 5}$ & 111.4 \\
\hline $\mathbf{2}$ & $\mathbf{2 8 7 . 8}$ & 101.3 & $\mathbf{2 8 9 . 8}$ & 75.28 \\
\hline
\end{tabular}

To confirm these results as well as to look for any interactions between mode, book and piece type, we also ran a three-way ANOVA on the APB and APM measures. With APB we found a marginal main effect for mode, $F(1,95)=2.60, p=.111$, a main effect for piece type, $F(1,95)=6.82, p=.011$, and a significant interaction of mode and book, $F(1,95)=9.37, p=.003$. With APM we also found a main effect for mode, $F(1,79)=9.57, p=.003$, piece type, $F(1,79)=8.93, p=.004$, and the interaction of mode and book, $F(1,79)=10.14, p=.002$. The main effect for piece type is expected and irrelevant to our interests here. What is important is that there is no effect for the interaction of mode and piece type. The main effect for mode as well as for the interaction of mode and book in both the APB and APM measures is consistent with our overall findings reported here.

Though we did not initially plan to look at the BPM directly, it was convenient to run the numbers. Thus, Table 3 shows the average BPM suggested for minor and major pieces in books one and two. Again, in order to avoid violating the assumption of independence, we averaged the suggested BPM for each piece before running the ANOVA. Though we found a trend in the direction of our hypothesis (minor is slower than major), this difference was not significant. Oddly, there is also a non-significant trend suggesting that the second book is interpreted by the editors as slower than the first book. A three way ANOVA for BPM found no significant interactions or main effects of mode, book, or piece (though mode was the closest to being significant at $F[1,79]=2.01, p=0.160)$.

Table 3. Average Beats Per Minute (BPM) for major and minor pieces of books 1 and 2 with combined editors.

\begin{tabular}{|l|l|l|l|l|}
\hline Book & Major & Stand. Dev. & Minor & Stand. Dev. \\
\hline $\mathbf{1}$ & $\mathbf{8 6 . 0 9}$ & 17.80 & $\mathbf{8 0 . 1 2}$ & 19.69 \\
\hline $\mathbf{2}$ & $\mathbf{8 0 . 9 6}$ & 23.14 & $\mathbf{7 4 . 2 3}$ & 17.38 \\
\hline
\end{tabular}

Figure 2 shows the percent distribution of time signatures for books 1 and 2 . We see that in book 1 all of the pieces with the half-note as the beat (-/2), an ostensibly 'slower' time signature (Palmer, 1981), 
were written in minor while all of the pieces with the sixteenth note as the beat (-/16), a 'faster' time signature, were in major. Furthermore over $70 \%$ of pieces with the eighth note as the beat (-/8), also a 'faster' time signature, were in major. A chi-squared test shows these findings to be statistically significant $\chi^{2}(d f=3, \mathrm{~N}=48)=7.26, p=.064$. It is important to note, however, that there are only 3 pieces in book 1 with a $-/ 2$ time signature and only 2 pieces in book one with a 16 . Nevertheless, we still find the results interesting, especially because they do not carry over to book 2 . The 'slower' and 'faster' time signatures of book 2 are much more evenly distributed between major and minor pieces. Needless to say, what little differences that do show up are not statistically significant.

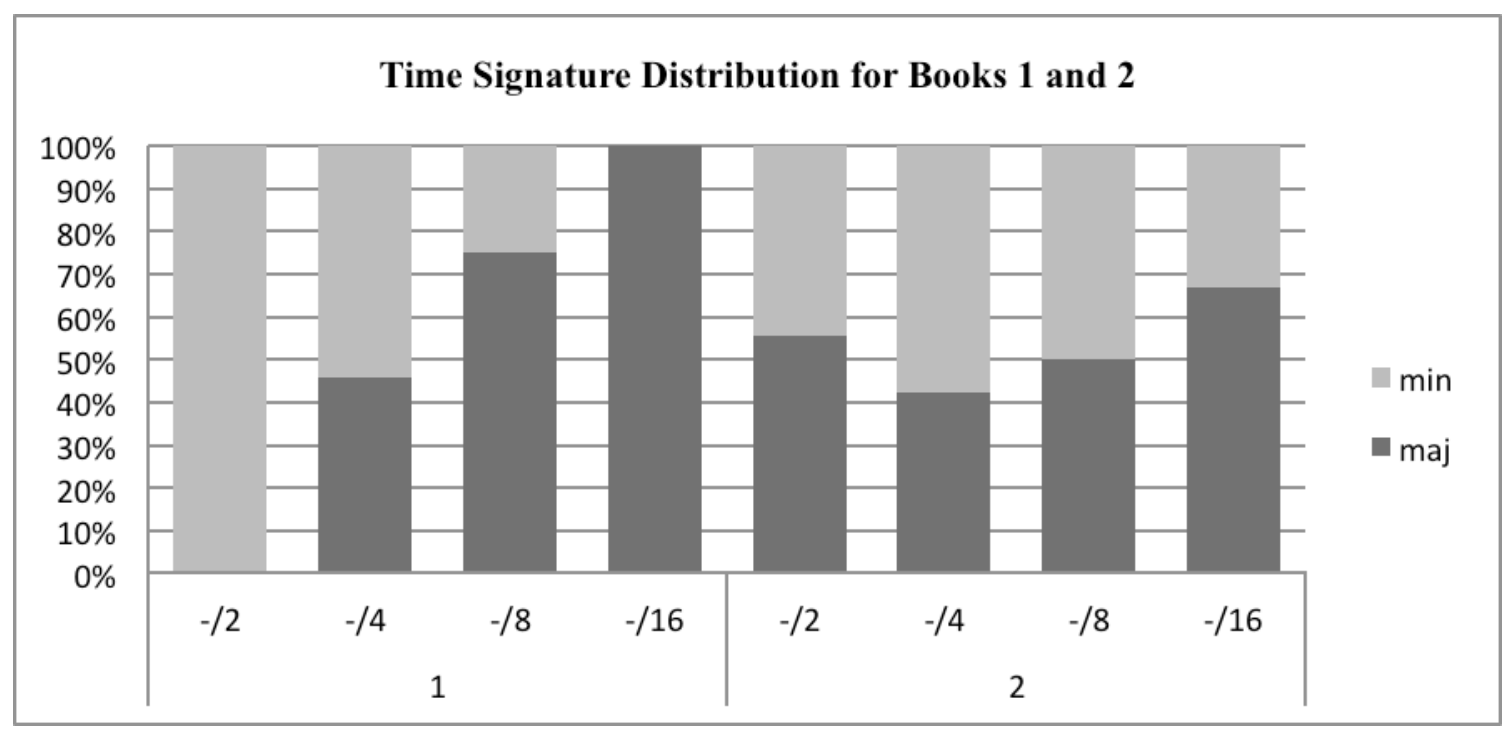

Fig. 2. Distribution of time signatures in books 1 and 2.

Figure 3 shows the distribution of tempo descriptions given by all of the editors. The results show that $80 \%$ of all slow descriptions in book 1 were given to minor pieces and over $70 \%$ of all fast descriptions in book 1 were given to major pieces. A chi-squared test shows these results to be statistically significant $\chi^{2}(d f=1, \mathrm{~N}=149)=40.70, p=.000$. The slow and fast descriptions from book 2 , however, were distributed nearly 50/50 to major and minor pieces and this difference was not significant. The omitted category of moderate/unclear tempo descriptions featured roughly the same proportion for both major mode and minor mode pieces in both books.

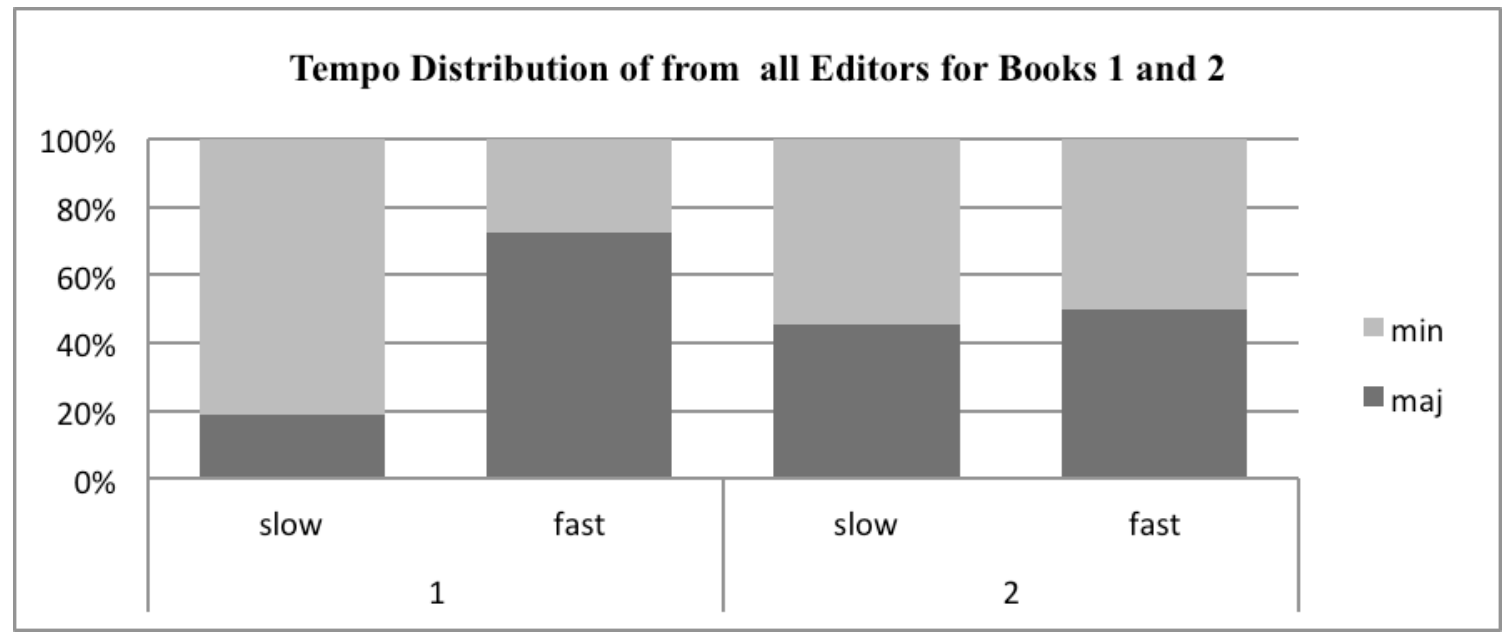

Fig. 3. Distribution of the tempo descriptions of all editors for books 1 and 2. 
As was suggested in the three-way ANOVA, if all of these results are recalculated with books 1 and 2 combined in order to compare all major pieces to all minor pieces, we do find a predominance of fast/major and slow/minor pairings that, with a few exceptions is still statistically significant. These results are shown in Table 4 and Figures 4 and 5: BPM and time signature distribution were not significant; APB was marginally significant at $F(1,95)=2.32, p=.131$; APM was significant at $F(1,79)=9.47, p=.003$; and tempo descriptions were significant at $\chi^{2}(d f=1 \mathrm{~N}=278)=25.21, p=.000$.

Table 4. APB and APM for books 1 and 2 combined.

\begin{tabular}{|l|l|l|l|l|}
\hline Books 1 \& 2 & Major & Stand. Dev. & Minor & Stand. Dev. \\
\hline APB & $\mathbf{4 . 0 3}$ & 1.15 & $\mathbf{3 . 6 9}$ & 1.07 \\
\hline APM & $\mathbf{3 3 9 . 3}$ & 116.9 & $\mathbf{2 6 5 . 1}$ & 98.9 \\
\hline BPM & $\mathbf{8 3 . 6 6}$ & 20.38 & $\mathbf{7 7 . 6 0}$ & 18.74 \\
\hline
\end{tabular}

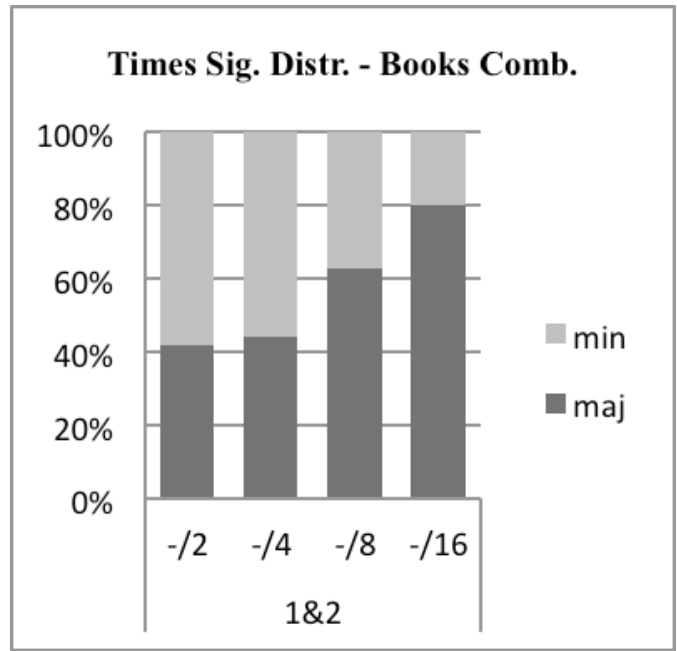

Fig. 4. Distribution of time signatures with books combined.

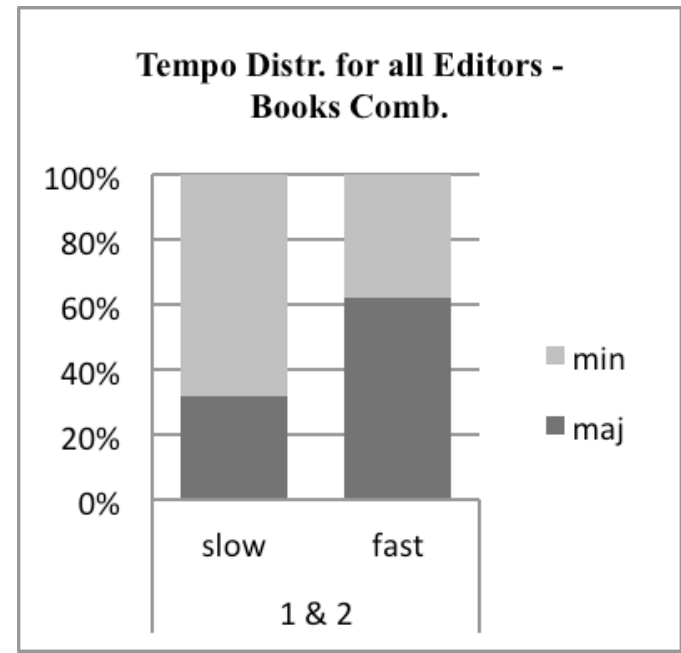

Fig. 5. Distribution of the tempo descriptions both for all editors with both books combined.

\section{DISCUSSION}

The results of this study are generally consistent with the hypothesis that there is a correlation between slow tempo and minor mode as well as between fast tempo and major mode in Bach's Well Tempered Clavier (WTC). This is also consistent with Post and Huron's (2009) finding that these pairings were predominant in Baroque and Classical music. However, this study also found that these tempo/mode pairings were only significantly present in Bach's WTC book 1 but not for his book 2.

Turner and Huron (2008) did a similar study to the one executed here where they looked at dynamic/mode pairings based on research in speech prosody that showed a correlation between quiet volume and sad emotion. They looked at prelude and fugue sets, as well, but they did a random sampling of several sets of preludes and fugues by various composers from different eras of Western classical music. They found that minor pieces were marked as significantly softer than major pieces.

Both Post and Huron (2009) as well as Turner and Huron (2008) were looking for slow/minor pairings and quiet/minor pairings as a means of bolstering the general notion that the minor mode corresponds to a sad emotion. This notion is supported by the work of Hevner (1935) and Gagnon (2003) among others. The minor mode cannot necessarily be equated with sadness, though. Post and Huron (2009) and Turner and Huron (2008) both discuss the fact that when the minor mode is paired with other features such as a fast tempo or loud dynamic, its association with a sad emotion is weakened. It is also important to 
consider that these pairings, at least in their original conception, may have had less to do with emotional character and more to do with a composer's stylistic or topical choices. For example, the fact that all of the time signatures with a half-note as the beat in book 1 were used in minor-mode pieces may be a result of the association of both the minor mode and the alle breve time signature with an old-fashioned church style or stile antico rather than an attempt on Bach's part to portray a sad emotion. However, just because Bach may not have intended such a combination to indicate a sad emotion does not mean that listeners (or at least modern listeners) may not hear it as such.

Unfortunately, we are unable to speculate as to why slow/minor and fast/major associations are found in the first book and not the second. Perhaps one of the more interesting outcomes of this study is that APM yielded a more robust operationalization of tempo than BMP. Furthermore, in attempting to better understand the difference between BPM and APM, we calculated the correlation between the BPM for each piece and the category (fast or slow) of its tempo description given by the corresponding editor. We then did the same thing for the APM of each piece. While neither correlation was very high, APM was higher than BPM $(r=.66$ and $r=.46$, respectively).

This low correlation found between tempo descriptions and metronome markings given by editors, even when subdivisions are included in the equation, is another intriguing finding. Our categorization of the tempo descriptions may have contributed somewhat to this lack of correlation. We included andante in the slow category and allegretto in the fast category but one could easily argue that andante and allegretto should both be included in the moderate/unclear category and omitted from the study. Nonetheless, it might be interesting to look more closely at the relationship between metronome markings and tempo descriptions by various editors to see what we can learn about how we perceive tempo in music. It might also be useful to do a historical study that looks at the use of certain tempo or character descriptions in music throughout time. This kind of historical study could give some insight to if and how perception of tempo has changed over time.

\section{NOTES}

[1] Please direct all correspondence to the first author at horn.232@osu.edu

[2] We could have found the same resulting APM by converting the editor's BPM to represent the quarternote.

\section{REFERENCES}

Bach, J.S., Ed. Bartok, B. (1964). Das Wohltemperierte Klavier. 1 \& 2. Boosey and Hawkes, Inc.

Bach, J.S., Ed. Bischoff, H. (1942). Das Wohltemperierte Klavier. 1 \& 2. Kalmus.

Bach, J.S., Ed. Irmer. (1950). Das Wohltemperierte Klavier. Teill 1 \& 2. Henle.

Bach, J.S., Ed. Mugellini, B. (1936). Das Wohltemperierte Klavier. 1 \& 2. Breitkopf.

Bach, J.S., Ed. Palmer, W. (1981). Well Tempered Clavier. Book 1. Alfred.

Bach, J.S., Ed. Tovey, D. (1924). Forty-Eight Preludes and Fugues. Book 1 \& 2. Associated Board.

Dalla Bella, S., Peretz, I., \& Rousseau L. (2001). A developmental study of the affective value of tempo and mode in music. Cognition, Vol. 80, B1-B10.

Desroches, R. L. (1997). Children's emotional response to the musical structures tempo, dynamics, and mode. Dissertation Abstracts International: Section B: The Sciences and Engineering, Vol. 59, 1882. 
Flowers, P. J. (1988). The effects of teaching and learning experiences, tempo, and mode on undergraduates' and children's symphonic music preferences. Journal of Research in Music Education, Vol. 36, No. 1, pp. 19-34.

Gagnon, L., \& Peretz, I. (2003). Mode and tempo relative contributions to 'happy-sad' judgements in equitone melodies. Cognition \& Emotion, Vol. 17, No. 1, pp. 25-40.

Hevner, K. (1935). The affective character of the major and minor modes in music. American Journal of Psychology, Vol. 47, No. 1, pp. 103-118.

Hevner, K. (1937). The affective value of pitch and tempo in music. American Journal of Psychology, Vol. 49, No. 4, pp. 621-630.

Husain, G., Thompson, W. F., \& Schellenberg, E. Glenn (2002). Effects of musical tempo and mode on arousal, mood, and spatial abilities. Music Perception, Vol. 20, No. 2, pp. 151-171.

LeBlanc, A., \& McCrary, J., (1983). Effects of tempo on children's music preference. Journal of Research in Music Education, Vol. 31, No. 4, pp. 283-294.

LeBlanc, A., Colman,J., McCrary, J., Sherrill, C., \& Mahlin, S. (1988). Tempo Preferences of different age music listeners. Journal of Research in Music Education, Vol. 36, No 3, pp. 156-168.

London, J. (2004). Hearing in Time. Oxford: Oxford University Press.

London, J. (2011). Tactus $\neq$ tempo: Some dissociations between attentional focus, motor behavior, and tempo judgment. Empirical Musicology Review, Vol. 6, No. 1, pp. 43-55.

Post, O., \& Huron, D. (2009). Western Classical Music in the Minor Mode is Slower (Except in the Romantic Period). Empirical Musicology Review, Vol. 4, No. 1, pp. 2-10.

Turner, B., \& Huron, D. (2008). A Comparison of Dynamics in Major- and Minor-key Works. Empirical Musicology Review, Vol. 3, No. 2, pp. 64-68. 